\title{
Prognostic value of spleen tyrosine kinase in human solid tumors
}

This article was published in the following Dove Press journal:

OncoTargets and Therapy

\author{
Beibei $\mathrm{Ni}^{1,2}$ \\ Shi $\mathrm{Li}^{1,2}$ \\ Yang Liu',2 \\ Yuqian Huang ${ }^{1,2}$ \\ Zesong $\mathrm{Li}^{1,2}$ \\ 'Guangdong Key Laboratory of \\ Systems Biology and Synthetic Biology \\ for Urogenital Tumors, First Affiliated \\ Hospital of Shenzhen University, \\ Shenzhen Second People's Hospital, \\ Shenzhen, Guangdong, People's \\ Republic of China; ${ }^{2}$ Shenzhen Key \\ Laboratory of Genitourinary Tumor, \\ First Affiliated Hospital of Shenzhen \\ University, Shenzhen Second People's \\ Hospital, Shenzhen, Guangdong, \\ People's Republic of China
}

Background: Spleen tyrosine kinase (SYK) was reported to be dysregulated in solid tumors and played an important role in cancer progression. However, the clinical and prognostic values of SYK in solid tumors remain unclear. This meta-analysis investigated the association between SYK expression and clinical outcomes in the patients with solid tumors.

Methods: A comprehensive literature search was conducted by screening the online electronic databases of PubMed, Embase, and the China National Knowledge Infrastructure. The hazard ratio (HR) with its corresponding 95\% CI was used to explore the prognostic value of SYK.

Results: We analyzed a total of 1,075 patients from 10 studies, which met the criteria for this meta-analysis. Our pooled results demonstrated that a low expression of SYK did not correlate significantly with shorter overall survival (OS; HR=0.64, 95\% CI: $0.34-1.21, P=0.169$ ) or poorer disease-free survival ( $\mathrm{HR}=0.51,95 \% \mathrm{CI}: 0.13-2.02, P=0.338)$. However, in a subgroup analysis based on tumor type and test method, under-expression of SYK was positively associated with worse OS in the groups of breast cancer (BC; HR=0.51, 95\% CI: $0.32-0.80, P=0.003$ ), hepatocellular carcinoma $(\mathrm{HCC}$; $\mathrm{HR}=0.44,95 \% \mathrm{CI}: 0.29-0.69, P<0.001)$, methylation $(\mathrm{HR}=0.39$, 95\% CI: $0.30-0.51, P<0.001)$, and quantitative reverse transcription polymerase chain reaction (HR $=0.24,95 \%$ CI: $0.09-0.65, P=0.005$ ).

Conclusion: This meta-analysis demonstrated that under-expression of SYK may serve as a predictive biomarker for poor prognosis in $\mathrm{BC}$ and $\mathrm{HCC}$ patients. In other solid tumors, the clinical usefulness should be confirmed by large-scale studies.

Keywords: spleen tyrosine kinase, solid tumor, prognosis, meta-analysis

\section{Introduction}

Cancer is one of the leading causes of morbidity and mortality worldwide. ${ }^{1}$ With the recent advances in precision medicine, the subclassification of cancer according to genotype, mutations, or biomarkers became the guidance in the selection of cancer treatments and the factor in prognosis. ${ }^{2}$ It is of great importance to investigate the potential biomarkers in cancers for both diagnostic and prognostic purposes.

The spleen tyrosine kinase (SYK) is a non-receptor tyrosine kinase that is expressed in most hematopoietic cells and functions as a hematopoietic cell-specific signaling molecule. ${ }^{3,4}$ SYK was first found to be a potential tumor suppressor in breast carcinoma in $2000 .{ }^{5}$ Since then, a growing amount of researches on SYK has been performed in non-hematopoietic tumors. ${ }^{6-8}$ Several clinical observations have indicated that patients with a low SYK expression have a significantly poorer prognosis than those with a high SYK expression. ${ }^{9-12}$ Functional studies have shown that overexpression of SYK is associated with cancer proliferation and metastasis. ${ }^{13-15}$ Epigenetic silencing through hypermethylation of promoter $\mathrm{CpG}$ islands has been proposed to be involved in the loss
Guangdong Key Laboratory of Systems Biology and Synthetic Biology for Urogenital Tumors, First Affiliated Hospital of Shenzhen University, Shenzhen Second People's Hospital, Shenzhen 5I8035, Guangdong, People's Republic of China Email lzssc@yahoo.com

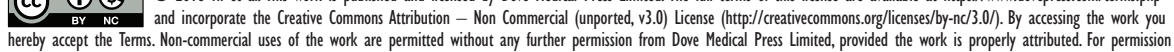
for commercial use of this work, please see paragraphs 4.2 and 5 of our Terms (https://www.dovepress.com/terms.php). 
of SYK expression in various cancers. ${ }^{16-19}$ Yang et a ${ }^{12}$ have reported that the expression level of SYK in tumor samples with unmethylated SYK gene was significantly higher than that in samples with methylated SYK gene. What is more, SYK methylation is correlated with poor overall survival (OS) in colorectal cancer (CRC). These data implicate SYK as a putative tumor suppressor in cancer. Contradictorily, recent studies have shown that SYK is upregulated in multiple types of tumors, including nasopharyngeal carcinoma (NPC), ${ }^{20}$ small-cell lung cancer, ${ }^{21}$ and squamous cell carcinoma of the head and neck cancer (SCCHN), ${ }^{22}$ suggesting that SYK may serve as a proto-oncogene. Taken together, we suspect that SYK played complicated roles in multiple cancer types.

Nevertheless, the prognostic value of SYK in cancer has not yet been fully elucidated. No meta-analysis has been conducted to assess the association between SYK and the survival of patients with cancers so far. Therefore, we performed a meta-analysis to assess the prognostic value of SYK in patients with various solid tumors. OS and diseasefree survival (DFS) were the primary end points.

\section{Methods}

\section{Search strategies}

The present study performed a thorough search for available literatures in databases of PubMed, Embase, and the China National Knowledge Infrastructure (CNKI) from inception up to August 2017. Search terms included "spleen tyrosine kinase", "SYK”, or "P72-Syk"; “cancer”, "tumor", "carcinoma", or "neoplasms"; or "prognostic", "prognosis", "survival", "mortality", "recurrence", or "outcome". In addition, the references of eligible studies, relevant systematic reviews, and meta-analyses in this field were manually retrieved. Two of the authors carried out this procedure independently, and any discrepancy was resolved by discussion.

\section{Study selection}

The inclusion criteria for the studies were as follows: 1) studies exploring any of the solid tumors; 2) assessing the association of SYK with OS and/or DFS; and 3) reporting sufficient information to estimate the hazard ratio (HR) and 95\% CI. The exclusion criteria were as follows: 1) studies on myelomas, lymphomas, or leukemia; 2) reviews, letters, case reports, and conference abstracts; 3) lacking essential information for calculating an $\mathrm{HR}$ and $95 \% \mathrm{CI}$; 4) overlapping or duplicate data; 5) laboratory studies on cell lines or animals level; and 6) studies with a sample size of $<20$ participants.

\section{Data extraction}

All eligible publications were reviewed by $\mathrm{Ni}$ and $\mathrm{Li}$. The following information was collected: the first author's name, country, year of publication, cancer type, number of patients, tumor stage, SYK test method, and outcome measures (OS and DFS). When HRs and their 95\% CIs were given in the articles, these data were extracted directly. If the prognosis was plotted as Kaplan-Meier (K-M) curve, the data were digitized by the software Engauge Digitizer version 4.1 and calculated as described. ${ }^{26}$

\section{Quality assessment}

The Newcastle-Ottawa Scale (NOS) was adopted to evaluate the quality of each eligible article. ${ }^{24}$ The NOS criterion was scored based on three aspects: 1) subject selection; 2) comparability of subject; and 3) clinical outcome of three categories. A total of nine items were extracted, and each item scored 1 . The total scores ranged from 0 to 9 . Scores of 1-3, 4-6, and 7-9 were defined as low-, intermediate-, and high-quality studies, respectively.

\section{Statistical analyses}

The meta-analysis was conducted using Stata 12.0 software (StataCorp LP, College Station, TX, USA). For the quantitative aggregation of the survival results, HRs and their 95\% CIs were used. In studies where HRs and corresponding 95\% CIs were not directly reported, we estimated these values on the basis of available data, such as survival curves, using the methods developed by Parmar et al, ${ }^{23}$ Williamson et al, ${ }^{25}$ and Tierney et al. ${ }^{26}$

Heterogeneity of the HR of each study was quantified using the chi-square-based $Q$-statistic test. The assumption of homogeneity was considered as invalid for $I^{2}>50 \%$ and $P<0.10$. When there was no statistically significant heterogeneity, we used the fixed-effects model for pooling the results; otherwise, the random-effects model was applied. The $\operatorname{logHR}$ and standard error were used for aggregation of the survival results. Publication bias was evaluated using funnel plots and Begg's and Egger's tests. ${ }^{27,28}$ All $P$-values were two sided, being statistically significant when $P$-value was $<0.05$.

\section{Results}

\section{Search results}

Our initial search retrieved 1,533 potentially relevant papers from PubMed, Embase and CNKI. A total of 1,070 relevant studies were identified after removing duplicated records. 
After the screening of titles and abstracts, 1,037 studies were excluded. In all, 33 relevant studies were selected for further evaluation. A total of 23 studies were excluded after reviewing the full article. Thus, 10 studies, comprising a total of 1,075 patients, were included in the quantitative

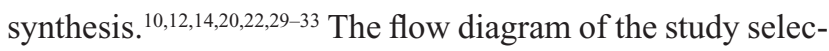
tion process is shown in Figure 1.

\section{Characteristics of the included studies}

The included studies were carried out in China ( $n=4)$, Japan $(n=2)$, Korea $(n=1)$, Canada $(n=1)$, Sweden $(n=1)$, and the UK $(n=1)$. The number of patients in each study varied from 32 to 226. Quantitative reverse transcription polymerase chain reaction (qRT-PCR) $(n=1)$, methylation $(n=3)$, and immunohistochemistry (IHC) $(n=6)$ were used to assess the SYK expression. The cutoff values varied across studies. The types of cancers involved in our study include SCCHN, $\mathrm{NPC}, \mathrm{CRC}$, hepatocellular carcinoma (HCC), gastric cancer (GC), oral squamous cell carcinoma (OSCC), non-small-cell lung cancer (NSCLC), and breast cancer (BC). There were nine studies for OS and three for DFS. HRs and 95\% CIs in five studies were extracted directly. HR values in five studies were extracted from $\mathrm{K}-\mathrm{M}$ survival curves. The features of the 10 studies that qualified for the present meta-analysis are summarized in Table 1.

\section{Meta-analysis: SYK expression, OS, and DFS in cancers}

Nine studies were included in the meta-analysis of OS. High heterogeneity among studies $\left(I^{2}=90.3 \%, P<0.001\right)$ was detected when nine studies were pooled. To make a conservative estimate, a random-effects model rather than a fixed-effects model was used to account for the highly significant inter-study heterogeneity. The results showed that a lower expression level of SYK did not correlate significantly with OS (HR=0.64, 95\% CI: 0.34-1.21, $P=0.169$; Figure 2). A meta-analysis of HRs for DFS was performed on three studies. With obvious statistical heterogeneity $\left(I^{2}=90.7 \%\right.$, $P<0.001$ ), a random-effects model was used to pool HRs. Based on these limited data, no significant difference was

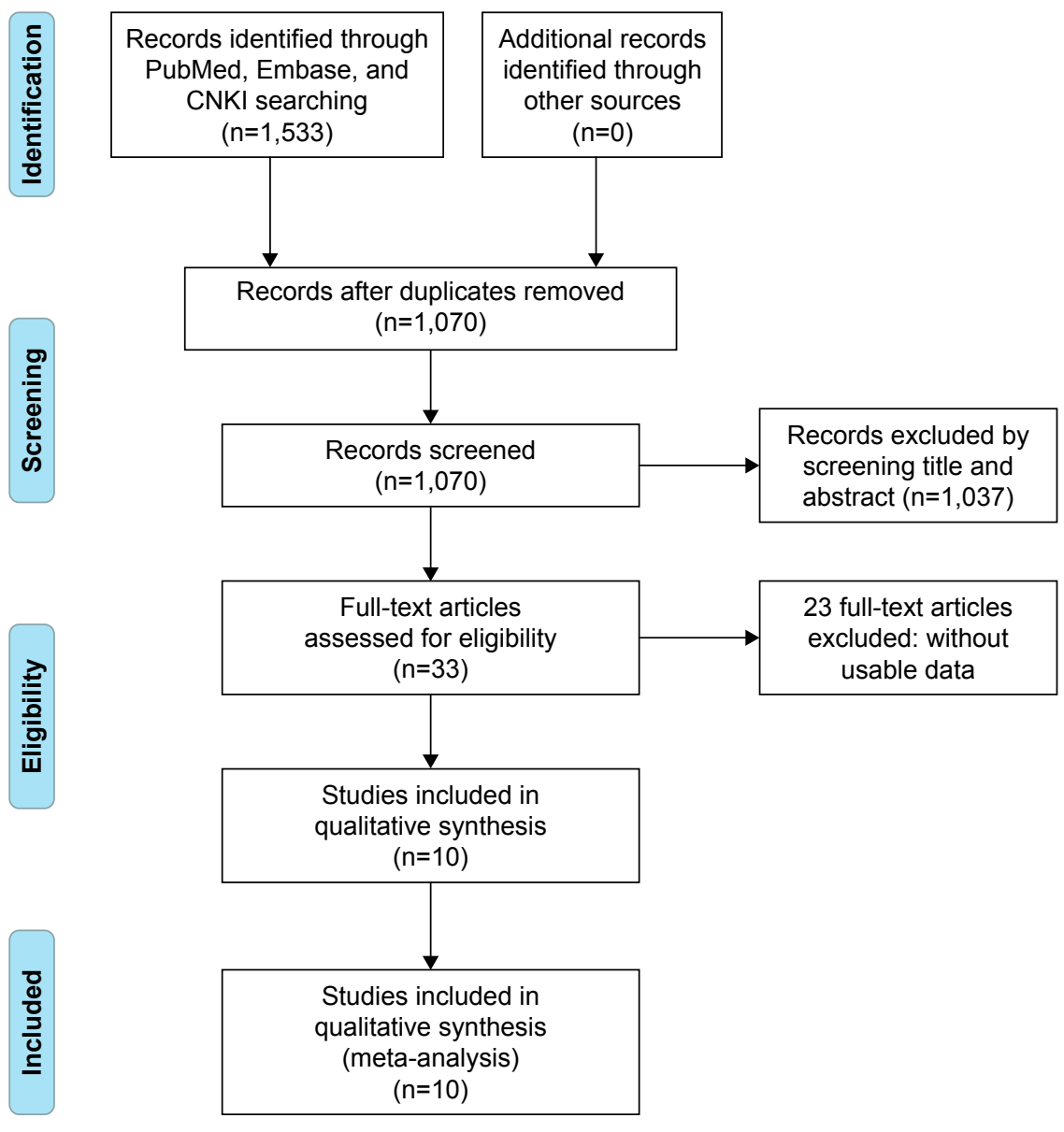

Figure I Flow diagram of study selection in present meta-analysis. Abbreviation: CNKI, China National Knowledge Infrastructure. 
Table I Characteristics of the studies included in the present meta-analysis

\begin{tabular}{|c|c|c|c|c|c|c|c|c|c|}
\hline Author & Region & Year & Cancer & Cases & $\begin{array}{l}\text { Tumor } \\
\text { stage }\end{array}$ & $\begin{array}{l}\text { Test } \\
\text { methods }\end{array}$ & $\begin{array}{l}\text { Outcome } \\
\text { indexes }\end{array}$ & HR (95\% Cl) & $\begin{array}{l}\text { NOS } \\
\text { score }\end{array}$ \\
\hline \multirow[t]{2}{*}{ Toyama et a ${ }^{29}$} & Japan & 2003 & $B C$ & 90 & Mixed & qRT-PCR & OS, DFS & OS: 0.24 (0.09-0.65) & 9 \\
\hline & & & & & & & & DFS: $0.24(0.09-0.62)$ & \\
\hline Dejmek et $\mathrm{al}^{30}$ & Sweden & 2005 & $\mathrm{BC}$ & 75 & Mixed & $\mathrm{IHC}$ & OS & $0.62(0.37-1.03)$ & 6 \\
\hline Nakashima et $\mathrm{al}^{10}$ & Japan & 2006 & GC & 110 & Mixed & $\mathrm{IHC}$ & OS & $0.72(0.26-1.96)$ & 8 \\
\hline Yuan et al ${ }^{14}$ & China & 2006 & $\mathrm{HCC}$ & 124 & Mixed & MSP & OS & $0.25(0.12-0.55)$ & 9 \\
\hline Luangdilok et $\mathrm{a}^{22}$ & UK & 2007 & $\mathrm{SCCHN}$ & 38 & Mixed & $\mathrm{IHC}$ & OS & $3.10(1.84-5.21)$ & 8 \\
\hline \multirow[t]{2}{*}{ Du et a ${ }^{20}$} & China & 2012 & NPC & 226 & Mixed & $\mathrm{IHC}$ & OS, DFS & OS: I.9I (1.27-2.88) & 8 \\
\hline & & & & & & & & DFS: 1.55 (1.13-2.13) & \\
\hline Yang et $\mathrm{a}^{12}$ & China & 2013 & CRC & 210 & Mixed & MSP & OS & $0.36(0.26-0.5 \mathrm{I})$ & 7 \\
\hline Shin et $\mathrm{al}^{31}$ & Korea & 2014 & $\mathrm{HCC}$ & 95 & Mixed & MethyLight & OS & $0.58(0.34-0.98)$ & 8 \\
\hline Fu et $\mathrm{al}^{32}$ & Canada & 2016 & oscc & 32 & Mixed & $\mathrm{IHC}$ & DFS & $0.30(0.12-0.76)$ & 7 \\
\hline $\mathrm{Gao}^{33}$ & China & 2016 & NSCLC & 75 & Mixed & $\mathrm{IHC}$ & OS & $0.34(0.07-1.56)$ & 5 \\
\hline
\end{tabular}

Abbreviations: HR, hazard ratio; NOS, Newcastle-Ottawa Quality Assessment Scale; BC, breast cancer; qRT-PCR, quantitative reverse transcription polymerase chain reaction; OS, overall survival; DFS, disease-free survival; IHC, immunohistochemistry; GC, gastric cancer; HCC, hepatocellular carcinoma; MSP, methylation-specific polymerase chain reaction; SCCHN, squamous cell carcinoma of the head and neck; NPC, nasopharyngeal carcinoma; CRC, colorectal cancer; OSCC, oral squamous cell carcinoma; NSCLC, non-small-cell lung cancer.

observed in survival between SYK expression and DFS (HR=0.51, 95\% CI: 0.13-2.02, $P=0.338$; Figure 3).

Given that the substantial heterogeneity exhibited in the trials aggregated with respect to the OS, subgroup analyses were carried out to explore the heterogeneity of covariates including country, tumor type, test method, and extracting method. As indicated in Table 2, according to subgroups of different countries, both China group and non-China group showed no significant HR (China HR $=0.52,95 \%$ CI: $0.17-1.56$,
$P=0.243$; non-China HR $=0.75,95 \%$ CI: $0.32-1.74, P=0.503$, random-effects model). For the tumor type, the subgroups (HCC and BC) were explored, from which the lower SYK expression was identified as a worse prognostic marker (HCC HR $=0.44,95 \%$ CI: $0.29-0.69, I^{2}=67.3 \%, P<0.001$; BC HR $=0.51,95 \%$ CI: $0.32-0.80, I^{2}=64.2 \%, P=0.003$, fixed-effects model). Other cancers group showed no significant HR (HR=0.94, 95\% CI: 0.34-2.6, $l^{2}=93.8 \%, P=0.903$, random-effects model). In the subgroup analysis based on
Study ID

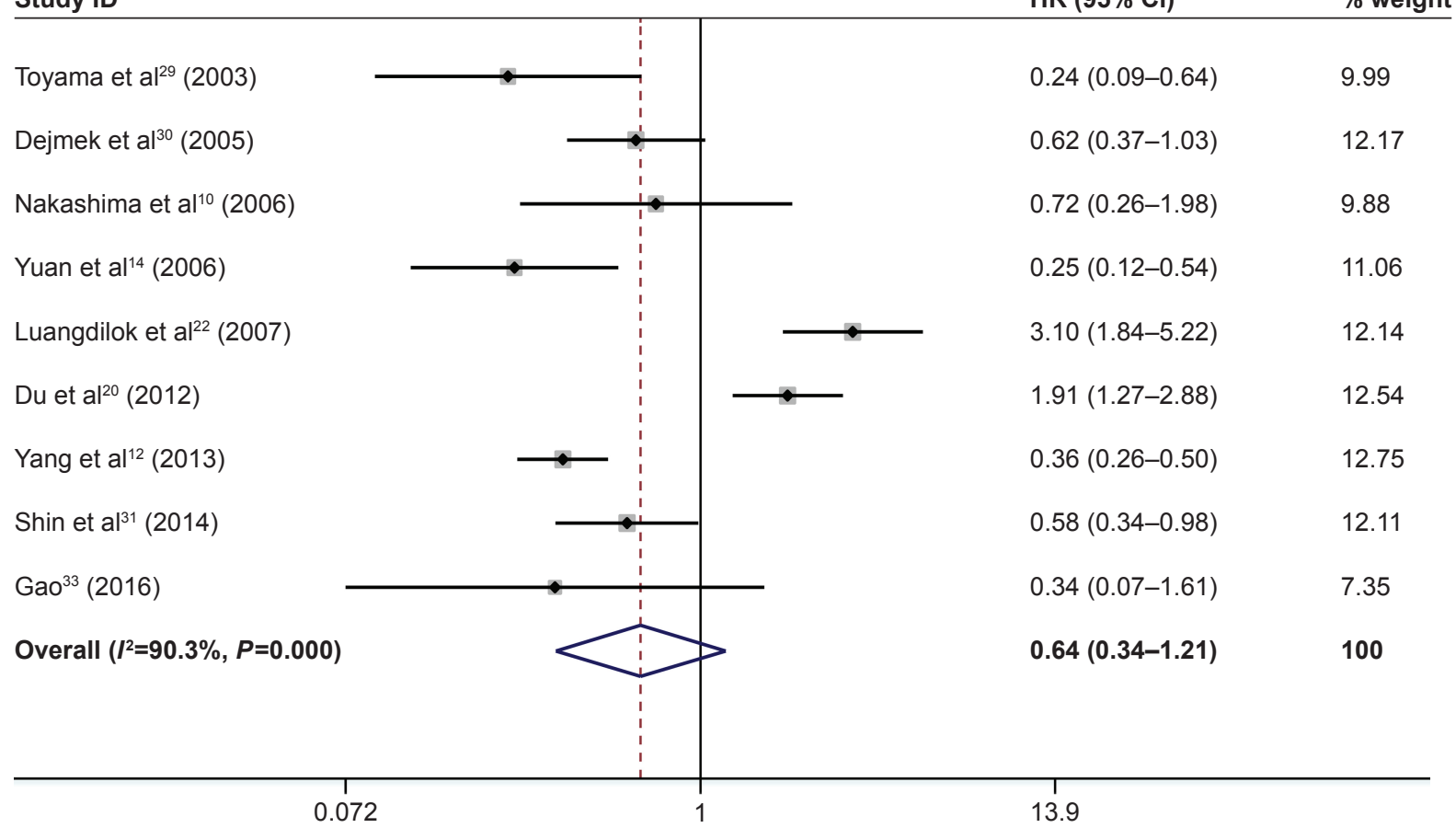

Figure 2 Forest plot of the relationship between SYK expression and OS in patients with solid tumors.

Note: Weights are from random-effects analysis.

Abbreviations: SYK, spleen tyrosine kinase; OS, overall survival; HR, hazard ratio. 


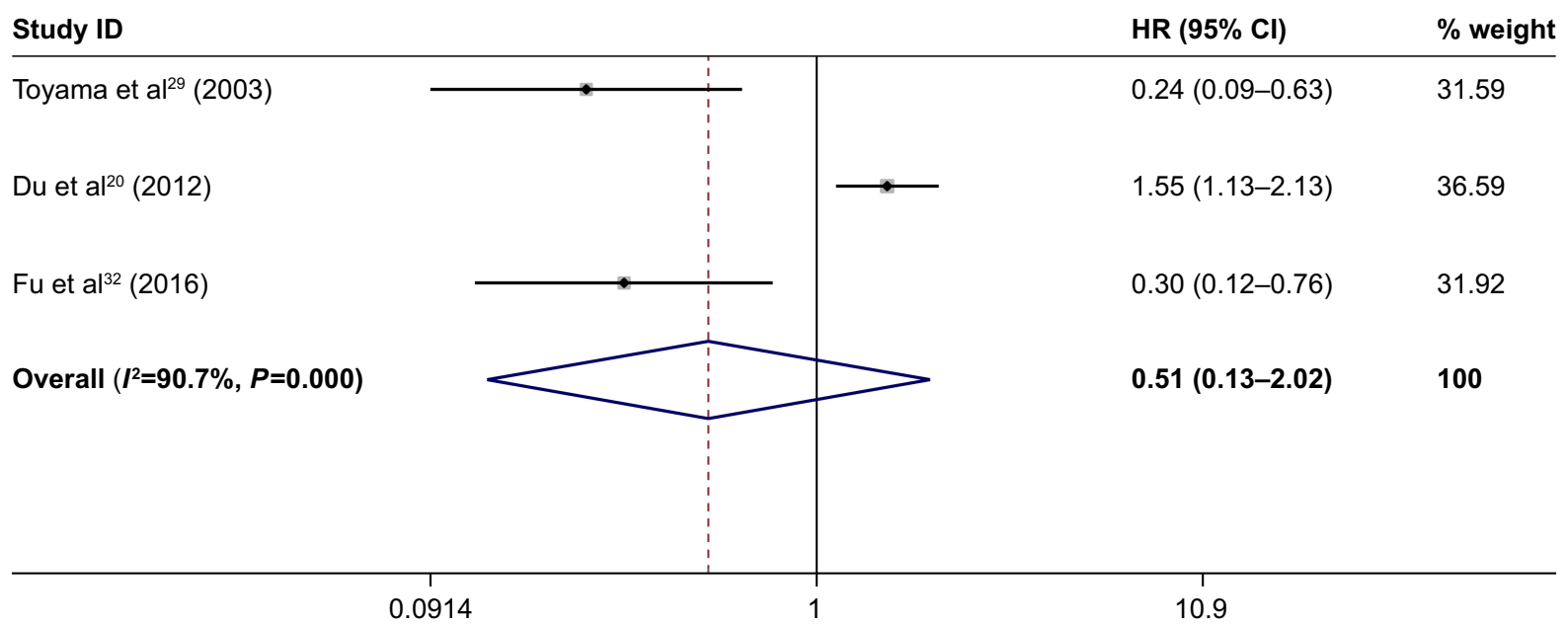

Figure 3 Forest plot of the relationship between SYK expression and DFS in solid tumors. Note: Weights are from random-effects analysis.

Abbreviations: SYK, spleen tyrosine kinase; DFS, disease-free survival; HR, hazard ratio.

test methods, the IHC group (HR=1.18, 95\% CI: $0.54-2.35$, $I^{2}=84.5 \%, P=0.75$, random-effects model) showed no significant HR, while the methylation group (HR $=0.39,95 \% \mathrm{CI}$ : $0.30-0.51, I^{2}=44.7 \%, P<0.001$, random-effects model) and the qRT-PCR group (HR=0.24, $95 \% \mathrm{CI}$ : $0.09-0.65, P=0.005$ ) showed significant HR. Similar to country-based subgroups analysis, both groups showed no significant HR in the subgroup analysis of extracting method.

\section{Publication bias}

The Begg's funnel plot and Egger's test were performed to evaluate the publication bias. As shown in Figure 4A,

Table 2 Subgroup analyses for associations between SYK expression and OS

\begin{tabular}{|c|c|c|c|c|c|}
\hline \multirow[t]{2}{*}{ Subgroup } & \multirow{2}{*}{$\begin{array}{l}\text { No of } \\
\text { studies }\end{array}$} & \multicolumn{2}{|c|}{ Heterogeneity } & \multirow[t]{2}{*}{$P$-value } & \multirow[t]{2}{*}{ HR (95\% Cl) } \\
\hline & & $I^{2}(\%)$ & $P$-value & & \\
\hline \multicolumn{6}{|l|}{ Country } \\
\hline China & 4 & 93.3 & 0.000 & 0.243 & $0.52(0.17-1.56)$ \\
\hline Non-China & 5 & 88.0 & 0.000 & 0.503 & $0.75(0.32-1.74)$ \\
\hline \multicolumn{6}{|l|}{ Tumor type } \\
\hline $\mathrm{HCC}$ & 2 & 67.3 & 0.080 & 0.000 & $0.44(0.29-0.69)$ \\
\hline $\mathrm{BC}$ & 2 & 64.2 & 0.095 & 0.003 & $0.5 \mathrm{I}(0.32-0.8)$ \\
\hline Other & 5 & 93.8 & 0.000 & 0.903 & $0.94(0.34-2.6)$ \\
\hline \multicolumn{6}{|l|}{ Test method } \\
\hline $\mathrm{IHC}$ & 5 & 84.5 & 0.000 & 0.750 & I. 18 (0.54-2.35) \\
\hline Methylation & 3 & 44.7 & 0.164 & 0.000 & $0.39(0.30-0.5 \mathrm{I})$ \\
\hline qRT-PCR & 1 & NA & NA & 0.005 & $0.24(0.09-0.65)$ \\
\hline \multicolumn{6}{|c|}{ Extracting method } \\
\hline K-M curve & 4 & 90.4 & 0.000 & 0.540 & $0.68(0.20-2.29)$ \\
\hline Report & 5 & 91.0 & 0.000 & 0.200 & $0.60(0.27-1.32)$ \\
\hline
\end{tabular}

Abbreviations: SYK, spleen tyrosine kinase; OS, overall survival; $\mathrm{HR}$, hazard ratio; $\mathrm{HCC}$, hepatocellular carcinoma; BC, breast cancer; IHC, immunohistochemistry; qRT-PCR, quantitative reverse transcription polymerase chain reaction; NA, not available; K-M, Kaplan-Meier. the shape of the funnel plot was relatively symmetrical and no publication bias was found in the OS meta-analysis (Begg's test, $P=0.917$; Egger's test, $P=0.698$ ). In the DFS meta-analysis, the Egger's test was significant $(P=0.024)$ for publication bias but the Begg's test was not $(P=0.296$; Figure 4B). Considering the abnormal distribution of the included patient numbers and the discrepancies of these three tests, the Egger test was not trusted. Therefore, there is no significant publication bias in the abovementioned analysis.

\section{Discussion}

To the best of our knowledge, no meta-analysis has been conducted to assess the possible prognostic role of SYK downregulation in solid malignancies before. To provide comprehensive and reliable conclusions, we conducted the present meta-analysis to assess the prognostic value of SYK in tumors.

The potential role of SYK in cancer prognosis has been extensively explored in various cancers. ${ }^{29-36}$ However, inconsistent results were found in different studies. . $^{10,12,20,22}$ More and more evidence has demonstrated that SYK acts as a tumor suppressor and is frequently downregulated in multiple types of cancer. In BC patient samples, Moroni et $\mathrm{al}^{37}$ found that the loss of SYK expression was progressive during tumor development and the low SYK levels were correlated with an increased risk of metastasis. In GC, loss of SYK expression was closely related to the malignant property of GC in the context of tumor depth and lymph node metastasis, especially in early GC. ${ }^{10}$ Similarly, Ogane et $\mathrm{al}^{8}$ found that SYK was downregulated in OSCC and correlated significantly with tumor metastasis to cervical lymph nodes. Bailet et al ${ }^{38}$ also demonstrated that SYK exerted its 

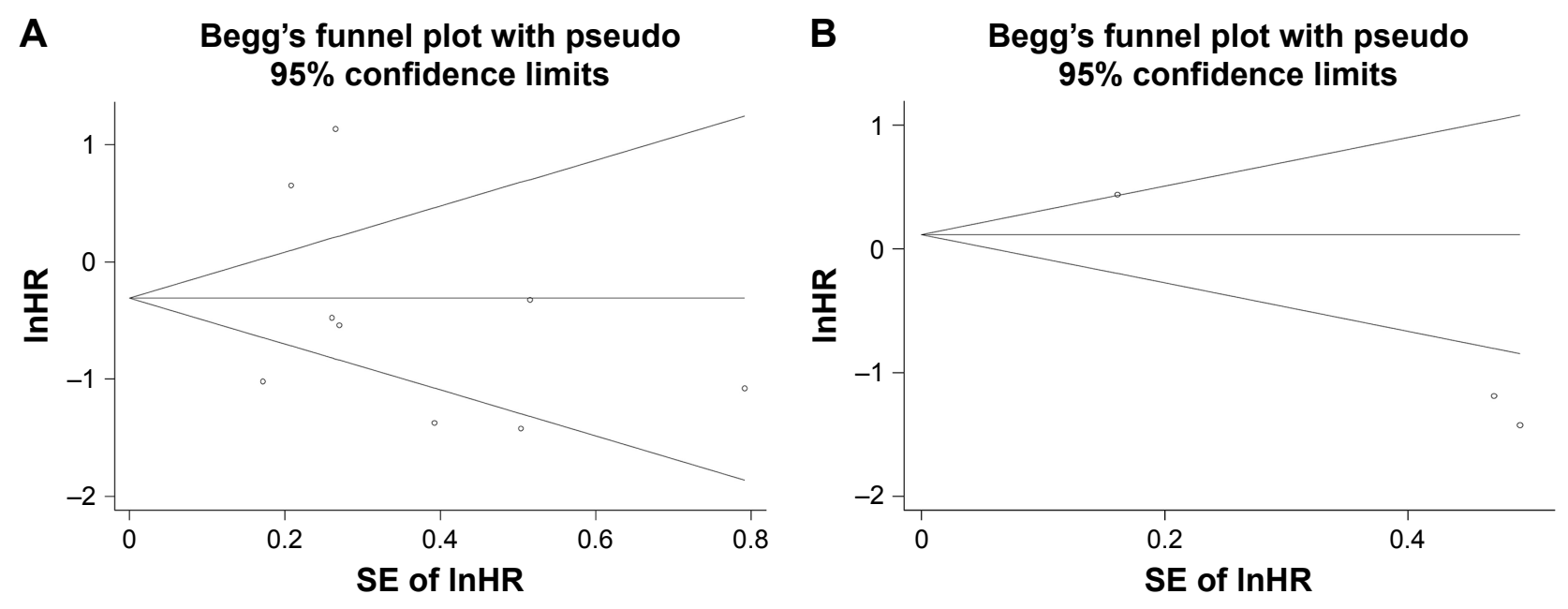

Figure 4 Funnel plot to evaluate potential publication bias of the included studies. Notes: (A) Funnel plot for nine studies reporting OS. (B) Funnel plot for three studies reporting DFS. Abbreviations: OS, overall survival; DFS, disease-free survival; SE, standard error; HR, hazard ratio.

antitumor activity through induction of a senescence-like growth arrest and activation of a p53-dependent pathway, suggesting that loss of SYK may contribute to the senescence bypass generally observed in malignant melanoma. Moreover, Yu et al ${ }^{39}$ observed that inhibition of SYK potentiated paclitaxel-induced cytotoxicity in ovarian cancer cells by stabilizing microtubules. In CRC, SYK(L) and SYK(S) could increase the cellular sensitivity to 5 -fluorouracil. ${ }^{40}$ On the other hand, in NPC, a high expression of SYK is associated with tumor recurrence and poor prognosis of patients. ${ }^{20}$ Udyavar et $\mathrm{al}^{21}$ reported that SYK was highly overexpressed in small-cell lung cancer tissues compared to normal tissues and SYK knockdown caused a significant decrease in proliferation rates compared to scrambled control in both H69 and H146. Further experiments need to be conducted to elucidate the role of SYK in carcinogenesis. However, among all the studies referring to the relationship between SYK and OS/DFS, there are still some contradictory results requiring adequate attention; a comprehensive study is therefore in urgent need.

Pooled analysis of 10 studies with 1,075 patients demonstrated no significant difference in survival between OS/DFS and SYK expression, which may partly be because of the small eligible number of studies and high heterogeneity. Subgroup analysis in OS was performed to explore the source of heterogeneity based on country, tumor type, test method, and extracting method. Based on the analysis and summary of existing literature, we found that under-expression of SYK is related to poorer OS in the qRT-PCR group and the methylation group, whereas there was no significant association between under-expression of SYK and OS in the IHC group. By analyzing the subgroup stratified by cancer type, we found that the magnitude effect of SYK on OS appeared to perform its best predicted effect on cancer patient's prognosis in BC and HCC. We suspected that the differences in SYK behavior in different cancer types may be due in part to unique pathogenic mechanisms in each cancer type and differences in the contribution of SYK to tumor biology.

Some limitations should be noted for this meta-analysis. First of all, despite the usage of random-effects model and subgroup analysis, the heterogeneity across studies failed to be eliminated completely, which could result in bias of the outcome to a certain extent. Second, some HRs and their corresponding 95\% CIs were estimated from $\mathrm{K}-\mathrm{M}$ survival curves, which may bring a slight error in HR and 95\% CI; hence, the prognostic value of SYK expression may be overestimated. Third, although we performed a comprehensive analysis and got significant conclusions, the general problems with meta-analysis, ie, limited number of studies, the heterogeneity among the studies, and so on, should be considered and verified in the future applications. Fourth, this study included different types of solid tumors to gain a general insight into the association between SYK expression and the clinical outcomes. However, different cancer types are likely to have different pathogenic mechanisms; therefore, SYK may play complicated roles in various cancers. Despite the limitations mentioned earlier, there are still numerous valuable implications in the current study. High-quality, large-sample, and multi-center prospective studies are preferable to confirm the findings of this study before the application of SYK for the prognosis of cancers. 


\section{Conclusion}

This systematic review and comprehensive meta-analysis demonstrates that a low expression of SYK plays an important role in human solid tumors.

\section{Acknowledgment}

This study was supported by grants from the Natural Science Foundation of Guangdong Province (No 2017A030310613), and the Foundation of Shenzhen Science Technology and Innovation Commission (No JCYJ20160427173722143).

\section{Disclosure}

The authors report no conflicts of interest in this work.

\section{References}

1. Siegel RL, Miller KD, Jemal A. Cancer statistics, 2018. CA Cancer J Clin. 2018;68:7-30.

2. Shukla HD. Comprehensive analysis of cancer-proteogenome to identify biomarkers for the early diagnosis and prognosis of cancer. Proteomes. 2017;5(4):E28.

3. Grädler U, Schwarz D, Dresing V, et al. Structural and biophysical characterization of the Syk activation switch. J Mol Biol. 2013 425(2):309-333.

4. Coopman PJ, Mueller SC. The spleen tyrosine kinase: a new negative regulator in tumor growth and progression. Cancer Lett. 2006; 241(2):159-173

5. Coopman PJ, Do MT, Barth M, et al. The Syk tyrosine kinase suppresses malignant growth of human breast cancer cells. Nature. 2000 406(6797):742-747.

6. Wang L, Devarajan E, He J, Reddy SP, Dai JL. Transcription repressor activity of spleen tyrosine kinase mediates breast tumor suppression. Cancer Res. 2005;65(22):10289-10297.

7. Zhang X, Shrikhande U, Alicie BM, Zhou Q, Geahlen RL. Role of the protein tyrosine kinase Syk in regulating cell-cell adhesion and motility in breast cancer cells. Mol Cancer Res. 2009;7(5):634-644.

8. Ogane S, Onda T, Takano N, Yajima T, Uchiyama T, Shibahara T. Spleen tyrosine kinase as a novel candidate tumor suppressor gene for human oral squamous cell carcinoma. Int J Cancer. 2009;124(11): 2651-2657.

9. Hong J, Hu K, Yuan Y, et al. CHK1 targets spleen tyrosine kinase (L) for proteolysis in hepatocellular carcinoma. J Clin Invest. 2012;122(6): 2165-2175.

10. Nakashima $\mathrm{H}$, Natsugoe $\mathrm{S}$, Ishigami $\mathrm{S}$, et al. Clinical significance of nuclear expression of spleen tyrosine kinase (Syk) in gastric cancer. Cancer Lett. 2006;236(1):89-94.

11. Peng C, Sun Q, Hao Y, Cong B, Zhao Y, Zhao X. Syk is low-expressed in non-small-cell lung cancer and inversely correlates with patient's survival. Acta Biochim Biophys Sin (Shanghai). 2013;45(2):149-151.

12. Yang $\mathrm{Z}$, Huo L, Chen H, et al. Hypermethylation and prognostic implication of Syk gene in human colorectal cancer. Med Oncol. 2013; 30(2):586.

13. Layton T, Stalens C, Gunderson F, Goodison S, Sillett S. Syk tyrosine kinase acts as a pancreatic adenocarcinoma tumor suppressor by regulating cellular growth and invasion. Am J Pathol. 2009;175(6): 2625-2636.

14. Yuan YF, Wang JP, Li JP, et al. Frequent epigenetic inactivation of spleen tyrosine kinase gene in human hepatocellular carcinoma Clin Cancer Res. 2006;12(22):6687-6695.

15. Peng C, Zhang Y, Sun Q, et al. Inhibitory effects of Syk transfection on lung cancer cell invasion. Asian Pac J Cancer Prev. 2013;14(5): 3001-3003.
16. Kunze E, Wendt M, Schlott T. Promoter hypermethylation of the 14-3-3 sigma, SYK and CAGE-1 genes is related to the various phenotypes of urinary bladder carcinomas and associated with progression of transitional cell carcinomas. Int J Mol Med. 2006;18(4):547-557.

17. Zhao S, Sun G, Tony PW, Ma D, Zhao C. Expression and methylation status of the Syk gene in cervical carcinoma. Arch Gynecol Obstet. 2011; 283(5):1113-1119.

18. Wang S, Ding YB, Chen GY, Xia JG, Wu ZY. Hypermethylation of Syk gene in promoter region associated with oncogenesis and metastasis of gastric carcinoma. World J Gastroenterol. 2004;10(12):1815-1818.

19. Hong H, Zhou K, Fu P, et al. Relationship between promoter methylation of Syk and Runx3 genes and postoperative recurrence and metastasis in gastric carcinoma. Zhonghua Zhong Liu Za Zhi. 2014;36(5): 341-345.

20. Du ZM, Kou CW, Wang HY, et al. Clinical significance of elevated spleen tyrosine kinase expression in nasopharyngeal carcinoma. Head Neck. 2012;34:1456-1464.

21. Udyavar AR, Hoeksema MD, Clark JE, et al. Co-expression network analysis identifies Spleen Tyrosine Kinase(SYK) as a candidate oncogenic driver in a subset of small-cell lung cancer. BMC Syst Biol. 2013;7(suppl 5):S1.

22. Luangdilok S, Box C, Patterson L, et al. Syk tyrosine kinase is linked to cell motility and progression in squamous cell carcinomas of the head and neck. Cancer Res. 2007;67(16):7907-7916.

23. Parmar MK, Torri V, Stewart L. Extracting summary statistics to perform meta-analyses of the published literature for survival endpoints. Stat Med. 1998;17(24):2815-2834.

24. Stang A. Critical evaluation of the Newcastle-Ottawa scale for the assessment of the quality of nonrandomized studies in meta-analyses. Eur J Epidemiol. 2010;25:603-605.

25. Williamson PR, Smith CT, Hutton JL, Marson AG. Aggregate data meta-analysis with time-to-event outcomes. Stat Med. 2002;21(22): 3337-3351.

26. Tierney JF, Stewart LA, Ghersi D, Burdett S, Sydes MR. Practical methods for incorporating summary time-to-event data into meta-analysis. Trials. 2007;8:16.

27. Egger M, Davey Smith G, Schneider M, Minder C. Bias in meta-analysis detected by a simple, graphical test. BMJ. 1997;315:629-634.

28. Begg CB, Mazumdar M. Operating characteristics of a rank correlation test for publication bias. Biometrics. 1994;50:1088-1101.

29. Toyama T, Iwase H, Yamashita H, et al. Reduced expression of the Syk gene is correlated with poor prognosis in human breast cancer. Cancer Lett. 2003;189(1):97-102.

30. Dejmek J, Leandersson K, Manjer J, et al. Expression and signaling activity of Wnt-5a/discoidin domain receptor-1 and Syk plays distinct but decisive roles in breast cancer patient survival. Clin Cancer Res. 2005; 11:520-528.

31. Shin SH, Lee KH, Kim BH, et al. Downregulation of spleen tyrosine kinase in hepatocellular carcinoma by promoter $\mathrm{CpG}$ island hypermethylation and its potential role in carcinogenesis. Lab Invest. 2014; 94(12):1396-1405.

32. Fu G, Somasundaram RT, Jessa F, et al. ER maleate is a novel anticancer agent in oral cancer: implications for cancer therapy. Oncotarget. 2016; 7(13):17162-17181.

33. Gao D. SYK(L) Interacts with YY1 and Coordinately Suppresses Slug-Mediated EMT in Non-Small Cell Lung Cancer Cells [Doctoral dissertation]. Tianjin: Tianjin Medical University; 2016.

34. Duan L, Ye L, Zhao G, et al. Serum spleen tyrosine kinase and vascular endothelial growth factor-C levels predict lymph node metastasis of oesophageal squamous cell carcinoma. Eur J Cardiothorac Surg. 2013; 43(3):e58-e63.

35. Ghotra VP, He S, van der Horst G, et al. SYK is a candidate kinase target for the treatment of advanced prostate cancer. Cancer Res. 2015; 75(1):230-240.

36. Hong J, Yuan Y, Wang J, et al. Expression of variant isoforms of the tyrosine kinase SYK determines the prognosis of hepatocellular carcinoma. Cancer Res. 2014;74(6):1845-1856. 
37. Moroni M, Soldatenkov V, Zhang L, et al. Progressive loss of Syk and abnormal proliferation in breast cancer cells. Cancer Res. 2004;64(20): 7346-7354.

38. Bailet O, Fenouille N, Abbe P, et al. Spleen tyrosine kinase functions as a tumor suppressor in melanoma cells by inducing senescence-like growth arrest. Cancer Res. 2009;69(7):2748-2756.
39. Yu Y, Gaillard S, Phillip JM, et al. Inhibition of spleen tyrosine kinase potentiates paclitaxel-induced cytotoxicity in ovarian cancer cells by stabilizing microtubules. Cancer Cell. 2015;28(1):82-96.

40. Ni BB, Hu J, Chen DK, et al. Alternative splicing of spleen tyrosine kinase differentially regulates colorectal cancer progression. Oncol Lett. 2016;12(3):1737-1744.

\section{Publish your work in this journal}

OncoTargets and Therapy is an international, peer-reviewed, open access journal focusing on the pathological basis of all cancers, potential targets for therapy and treatment protocols employed to improve the management of cancer patients. The journal also focuses on the impact of management programs and new therapeutic agents and protocols on

\section{Dovepress}

patient perspectives such as quality of life, adherence and satisfaction. The manuscript management system is completely online and includes a very quick and fair peer-review system, which is all easy to use. Visit http://www.dovepress.com/testimonials.php to read real quotes from published authors.

Submit your manuscript here: http://www.dovepress.com/oncotargets-and-therapy-journal 